\title{
RANK 2 WALL-CROSSING AND THE SERRE CORRESPONDENCE
}

\author{
AMIN GHOLAMPOUR AND MARTIJN KOOL
}

\begin{abstract}
We study Quot schemes of 0-dimensional quotients of sheaves on 3 -folds $X$. When the sheaf $\mathcal{R}$ is rank 2 and reflexive, we prove that the generating function of Euler characteristics of these Quot schemes is a power of the MacMahon function times a polynomial. This polynomial is itself the generating function of Euler characteristics of Quot schemes of a certain 0 -dimensional sheaf, which is supported on the locus where $\mathcal{R}$ is not locally free.

In the case $X=\mathbb{C}^{3}$ and $\mathcal{R}$ is equivariant, we use our result to prove an explicit product formula for the generating function. This formula was first found using localization techniques in previous joint work with B. Young. Our results follow from R. Hartshorne's Serre correspondence and a rank 2 version of a Hall algebra calculation by J. Stoppa and R. P. Thomas.
\end{abstract}

\section{INTRODUCTION}

Let $\mathcal{R}$ be a reflexive 1 sheaf on a smooth 3 -fold $X$. Since $X$ is 3 -dimensional, $\mathcal{R}$ is locally free outside a 0 -dimensional closed subset. For any torsion free sheaf $\mathcal{F}$ on $X$ its double dual $\mathcal{F}^{* *}$ is reflexive and we have a natural embedding $\mathcal{F} \hookrightarrow \mathcal{F}^{* *}$. Reflexive sheaves are much easier than torsion free sheaves, because they are determined by their restriction to the complement of any closed subset of codimension $\geq 2$ [Har2, Prop. 1.6].

Let $X=\mathbb{C}^{3}$ and consider the natural action of $T=\mathbb{C}^{* 3}$ on $X$. Suppose $\mathcal{R}$ is a rank $2 T$-equivariant reflexive sheaf on $X$. Then $H^{0}(\mathcal{R})$ is graded by the character group $X(T)=\mathbb{Z}^{3}$. We denote by $\operatorname{Quot}(\mathcal{R}, n)$ the Quot scheme of 0 -dimensional quotients $\mathcal{R} \rightarrow \mathcal{Q}$, where $\mathcal{Q}$ has length $n$. Using $T$-localization, the authors and B. Young calculated the topological Euler characteristics $e(\operatorname{Quot}(\mathcal{R}, n))$ GKY1]. The answer is as follows.

In the case $\mathcal{R}$ is locally free, it splits $T$-equivariantly as a sum of two line bundles [GKY1, Prop. 3.4]. The splitting gives an additional scaling action of $\mathbb{C}^{*}$ on the summands. It is not hard to show that localization with respect to

\footnotetext{
${ }^{1} \mathrm{~A}$ torsion free sheaf $\mathcal{F}$ is called reflexive if the natural embedding $\mathcal{F} \hookrightarrow \mathcal{F}^{* *}$ is an isomorphism. Here $(\cdot)^{*}:=\mathscr{H}$ om $\left(\cdot, \mathcal{O}_{X}\right)$ denotes the dual.
} 
$T \times \mathbb{C}^{*}$ gives

$$
\sum_{n=0}^{\infty} e(\operatorname{Quot}(\mathcal{R}, n)) q^{n}=M(q)^{2}
$$

where $M(q)=\prod_{k>0} 1 /\left(1-q^{k}\right)^{k}$ denotes the MacMahon function counting 3D partitions. The interesting case is when $\mathcal{R}$ is singular, i.e. not locally free. In this case the rank 2 module $H^{0}(\mathcal{R})$ has three homogeneous generators, whose weights can be uniquely written as

$$
\left(u_{1}, u_{2}, u_{3}\right)+\left(v_{1}, v_{2}, 0\right),\left(u_{1}, u_{2}, u_{3}\right)+\left(v_{1}, 0, v_{3}\right),\left(u_{1}, u_{2}, u_{3}\right)+\left(0, v_{2}, v_{3}\right),
$$

where $u_{1}, u_{2}, u_{3} \in \mathbb{Z}$ and $v_{1}, v_{2}, v_{3} \in \mathbb{Z}_{>0}$. See [GKY1, Prop. 3.4] for details.

Theorem 1.1 (Gholampour-Kool-Young). Let $\mathcal{R}$ be a singular rank $2 T$ equivariant reflexive sheaf on $\mathbb{C}^{3}$ with homogeneous generators of weights (11). Then

$$
\sum_{n=0}^{\infty} e(\operatorname{Quot}(\mathcal{R}, n)) q^{n}=M(q)^{2} \prod_{i=1}^{v_{1}} \prod_{j=1}^{v_{2}} \prod_{k=1}^{v_{3}} \frac{1-q^{i+j+k-1}}{1-q^{i+j+k-2}} .
$$

This formula was used as a building block for generating functions of Euler characteristics and virtual invariants of moduli spaces of rank 2 stable torsion free sheaves on toric 3-folds in [GKY1]. The RHS of this formula is $M(q)^{2}$ times the generating function of $3 \mathrm{D}$ partitions confined to the box $\left[0, v_{1}\right] \times\left[0, v_{2}\right] \times$ $\left[0, v_{3}\right][$ Sta, $(7.109)] 2$ The components of the fixed locus $\operatorname{Quot}(\mathcal{R}, n)^{T}$ are unions of products of $\mathbb{P}^{1}$ and the combinatorics of their enumeration is solved using the double dimer model in GKY2]. From the shape of the formula, we expected another proof by wall-crossing should exist, which prompted this paper. In this paper, we derive Theorem 1.1 from Theorem 1.2 below.

Let $X$ be any smooth projective 3 -fold and $\mathcal{R}$ a rank 2 reflexive sheaf on $X$. Then $\mathscr{E} x t^{1}\left(\mathcal{R}, \mathcal{O}_{X}\right)$ is a 0 -dimensional sheaf supported on the singularities of $\mathcal{R}$, i.e. on the locus where $\mathcal{R}$ is not locally free. The length of this sheaf is equal to $c_{3}(\mathcal{R})[$ Har2, Prop. 2.6].

Theorem 1.2. Let $\mathcal{R}$ be a rank 2 reflexive sheaf on a smooth projective 3fold $X$. Suppose $H^{1}(\operatorname{det}(\mathcal{R}))=H^{2}(\operatorname{det}(\mathcal{R}))=0$ and there exists a cosection $\mathcal{R} \rightarrow \mathcal{O}_{X}$ cutting out a 1-dimensional closed subscheme. Then

$$
\sum_{n=0}^{\infty} e(\operatorname{Quot}(\mathcal{R}, n)) q^{n}=M(q)^{2 e(X)} \sum_{n=0}^{\infty} e\left(\operatorname{Quot}\left(\mathscr{E} x t^{1}\left(\mathcal{R}, \mathcal{O}_{X}\right), n\right)\right) q^{n} .
$$

In particular the $R H S$ is $M(q)^{2 e(X)}$ times a polynomial of degree $c_{3}(\mathcal{R})$.

\footnotetext{
${ }^{2}$ More precisely: for each box of the 3D partition, the corner closest to the origin is an element of $\left[0, v_{1}-1\right] \times\left[0, v_{2}-1\right] \times\left[0, v_{3}-1\right]$.
} 
Remark 1.3. In an earlier version of this paper, we conjectured that (2) holds for any rank 2 reflexive sheaf $\mathcal{R}$ on any smooth projective threefold $X$. At the end of this introduction, we present an argument due to J. Rennemo, which indeed shows that this is the case. Essentially, after replacing $\mathcal{R}$ by an appropriate twist $\mathcal{R}(-m H)$, the vanishing conditions are automatically satisfied and the desired cosection always exists. Alternatively, in the sequel [GK2], we generalize (2) to arbitrary torsion free sheaves of homological dimension $\leq 1$ and any rank on any smooth projective threefold.

Remark 1.4 (Hilbert scheme version). Let $\mathcal{R}$ be a rank 2 reflexive sheaf on a smooth projective 3 -fold $X$. Since $\mathcal{R}$ is rank 2 and reflexive, it has homological dimension $\leq 1$ and admits a 2 -term resolution by vector bundles [Har2, Prop. 1.3]

$$
0 \rightarrow \mathcal{E}_{1} \rightarrow \mathcal{E}_{0} \rightarrow \mathcal{R} \rightarrow 0 .
$$

A priori we only know rk $\mathcal{E}_{0}-\mathrm{rk} \mathcal{E}_{1}=2$. However in the minimal rank case, i.e. $\mathrm{rk} \mathcal{E}_{1}=1$, we can dualize the above sequence and obtain

$$
\cdots \rightarrow \mathcal{E}_{1}^{*} \rightarrow \mathscr{E} x t^{1}\left(\mathcal{R}, \mathcal{O}_{X}\right),
$$

which shows $\mathscr{E} x t^{1}\left(\mathcal{R}, \mathcal{O}_{X}\right)$ is a structure sheaf (recall that $\mathscr{E} x t^{1}\left(\mathcal{R}, \mathcal{O}_{X}\right)$ is 0-dimensional). We denote the corresponding 0-dimensional subscheme by $\operatorname{Sing}(\mathcal{R})$. Then (2) implies

$$
\sum_{n=0}^{\infty} e(\operatorname{Quot}(\mathcal{R}, n)) q^{n}=M(q)^{2 e(X)} \sum_{n=0}^{\infty} e\left(\operatorname{Hilb}^{n}(\operatorname{Sing}(\mathcal{R}))\right) q^{n},
$$

where $\operatorname{Hilb}^{n}(\operatorname{Sing}(\mathcal{R}))$ denotes the Hilbert scheme of $n$ points on $\operatorname{Sing}(\mathcal{R})$. In particular the RHS is $M(q)^{2 e(X)}$ times a polynomial of degree $\ell(\operatorname{Sing}(\mathcal{R}))$, where $\ell(\cdot)$ denotes length. Theorem 1.1 is derived from (3) combined with a $T$-localization argument in Section 4.

1.1. Idea of proof and heuristics. The proof of Theorem 1.2 uses a rank 2 version of a wall-crossing (or rather Hall algebra) calculation by J. Stoppa and R. P. Thomas [ST] along the lines of [PT1, Section 3.3]. These Hall algebra methods were introduced by D. Joyce [Joy1, Joy2, Joy3, Joy4, and M. Kontsevich and Y. Soibelman [KS], see also [Bri1, Bri2]. We provide the main ideas leading to our formula:

(1) On one side of the wall (DT side), we are concerned with surjections 3 inside

$$
\operatorname{Ext}^{1}(\mathcal{R}, \mathcal{Q}[-1]) \cong \operatorname{Hom}(\mathcal{R}, \mathcal{Q})
$$

\footnotetext{
${ }^{3}$ Surjectivity can be regarded as a stability condition on the DT side.
} 
corresponding to short exact sequences

$$
0 \rightarrow \mathcal{F} \rightarrow \mathcal{R} \rightarrow \mathcal{Q} \rightarrow 0
$$

where $\mathcal{R}$ is fixed and $\mathcal{Q}$ varies among all 0-dimensional sheaves on $X$. Equivalenty, $\mathcal{F}$ varies over all rank 2 torsion free sheaves with reflexive hull $\mathcal{R}$ and 0 -dimensional cokernel. The sequence above gives the exact triangle

$$
\mathcal{Q}[-1] \rightarrow \mathcal{F} \rightarrow \mathcal{R}
$$

in the derived category $D^{b}(X)$. This is a rank 2 analog of (3.6) in PT1.

(2) On the other side of the wall (PT side), we obtain objects $A^{\bullet} \in D^{b}(X)$ given by 2 -term complexes of coherent sheaves that fit in exact triangles of the form

$$
\mathcal{R} \rightarrow A^{\bullet} \rightarrow \mathcal{Q}[-1]
$$

i.e. elements of $\operatorname{Ext}^{1}(\mathcal{Q}[-1], \mathcal{R})$. This exact triangle is an analog of $(3.7)$ in [PT1]. Next we use our assumption: the existence of a cosection $\mathcal{R} \rightarrow \mathcal{O}_{X}$ cutting out a 1 -dimensional closed subscheme $C \subset X$. This enables us to describe the objects $A^{\bullet}$ which are "stable" on the PT side of the wall. The cosection allows us to embed (Lemma 2.3)

$$
\operatorname{Ext}^{1}(\mathcal{Q}[-1], \mathcal{R}) \subset \operatorname{Ext}^{1}\left(\mathcal{Q}[-1], I_{C}\right) \cong \operatorname{Ext}^{1}\left(\mathcal{Q}, \mathcal{O}_{C}\right),
$$

where $I_{C} \subset \mathcal{O}_{X}$ denotes the ideal sheaf of $C \subset X$ and the second isomorphism follows from the usual short exact sequence. Note that elements of $\operatorname{Ext}^{1}\left(\mathcal{Q}, \mathcal{O}_{C}\right)$ correspond to short exact sequences

$$
0 \rightarrow \mathcal{O}_{C} \rightarrow F \rightarrow \mathcal{Q} \rightarrow 0 .
$$

The elements of $\operatorname{Ext}^{1}\left(\mathcal{Q}, \mathcal{O}_{C}\right)$ with purt $F$ are exactly the stable pairs (PT pairs) with underlying Cohen-Macaulay curve $C$. Therefore, inside $\operatorname{Ext}^{1}(\mathcal{Q}[-1], \mathcal{R})$ we have the locus of elements for which the corresponding extension in $\operatorname{Ext}^{1}\left(\mathcal{Q}, \mathcal{O}_{C}\right)$ is a PT pair. The objects $A^{\bullet}$ in this locus can be viewed as some kind of "rank 2 PT pairs". Note that this notion a priori depends on the choice of cosection $\mathcal{R} \rightarrow \mathcal{O}_{X}$.

(3) One of the key contributions of this paper is that we give a geometric description of the "stable objects" $A^{\bullet}$ of (2) purely in terms of the singularities of $\mathcal{R}$. At the level of generating functions this gets rid of the choice of cosection $\mathcal{R} \rightarrow \mathcal{O}_{X}$. Specifically, in Theorem [2.5, we prove that there exists a geometric bijection between the locus of "stable

\footnotetext{
${ }^{4}$ Purity can be regarded as a stability condition on the PT side.
} 
objects" $A^{\bullet}$ described in (2) and

$$
\bigsqcup_{n} \operatorname{Quot}\left(\mathscr{E} x t^{1}\left(\mathcal{R}, \mathcal{O}_{X}\right), n\right)
$$

The RHS of Theorem 1.2 can be seen as $M(q)^{2 e(X)}$ times the generating function of sub-PT pairs of a fixed PT pair, which we call the Hartshorne PT pair (or rather its dual). By the Serre correspondence, reviewed in Section 2.1. the data of a cosection $\mathcal{R} \rightarrow \mathcal{O}_{X}$ cutting out a 1-dimensional closed subscheme is equivalent to a Hartshorne PT pair. This is discussed in Remark 2.6.

Remark 1.5. [Toda] Very recently Y. Toda [Tod] developed a theory of higher rank PT pairs on any smooth projective Calabi-Yau 3-fold $X$. He proves a beautiful wall-crossing formula between the virtual counts of these pairs and higher rank Donaldson-Thomas invariants. The shape of his formula for rank 2 is similar to Theorem 1.2. This suggests our RHS can also be interpreted as $M(q)^{2 e(X)}$ times a generating function of rank 2 PT pairs (probably with several additional constraints). It is interesting to find out the precise relationship.

Acknowledgements. We thank R. P. Thomas for useful conversations. Special thanks go to B. Young. He originally found the product formula of Theorem 1.1 from the toric description of $\operatorname{Quot}(\mathcal{R}, n)^{T}$ using the double dimer model GKY1, GKY2]. His formula prompted this project. We thank the anonymous referee for pointing out why one needs $H^{2}(L)=0$ in the Hartshorne-Serre correspondence (Theorem 2.1). We also warmly thank J. Rennemo for pointing out that the assumptions of Theorem 1.2 can be weakened (Remark 1.3). A.G. was partially supported by NSF grant DMS-1406788. M.K. was supported by Marie Skłodowska-Curie Project 656898.

1.2. Rennemo's argument. Assume Theorem 1.2 holds. Then equation (2) holds for any rank 2 reflexive sheaf $\mathcal{R}$ on any smooth projective threefold $X$ (Remark 1.3). This follows from the following argument due to Rennemo 5 We may assume $\mathcal{R}$ is singular, because in the locally free case (2) easily follows by taking a trivialization of $\mathcal{R}$.

Let $H$ be a very ample divisor on $X$. Then equation (2) for $\mathcal{R}$ is equivalent to equation (2) for $\mathcal{R}^{\prime}:=\mathcal{R}(-m H)$, for any $m$. It suffices to show that for $m \gg 0, \mathcal{R}^{\prime}$ satisfies

- $H^{1}\left(\operatorname{det} \mathcal{R}^{\prime}\right)=H^{2}\left(\operatorname{det} \mathcal{R}^{\prime}\right)=0$, and

- there exists a cosection $\mathcal{R}^{\prime} \rightarrow \mathcal{O}_{X}$ cutting out a 1 -dimensional closed subscheme.

\footnotetext{
${ }^{5}$ We take full responsibility for the way in which the argument is presented.
} 
Since $\operatorname{det} \mathcal{R}^{\prime}=(\operatorname{det} \mathcal{R})(-2 m H)$, the first follows for $m \gg 0$ by Serre duality and Serre vanishing. Also $\mathcal{R}^{\prime *}=\mathcal{R}^{*}(m H)$ is globally generated for $m \gg 0$ :

$$
\pi: V \otimes \mathcal{O}_{X} \rightarrow \mathcal{R}^{\prime *},
$$

where $V:=H^{0}\left(X, \mathcal{R}^{\prime *}\right)$. Let $X^{\circ}=X \backslash \operatorname{Supp} \mathscr{E} x t^{1}\left(\mathcal{R}^{\prime}, \mathcal{O}_{X}\right)$, i.e. the complement of the finitely many points where $\mathcal{R}^{\prime}$ is singular, and let $N:=\operatorname{dim} V$. Restriction of (44) to points of $X^{\circ}$ induces a morphism

$$
f: X^{\circ} \rightarrow \mathrm{Gr}
$$

where $\operatorname{Gr}:=\operatorname{Gr}(N-2, V)$ denotes the Grassmannian of $(N-2)$-dimensional subspaces of $V$. Denote its universal quotient by $\Pi: V \otimes \mathcal{O}_{\mathrm{Gr}} \rightarrow \mathcal{Q}$, then $f^{*} \Pi=\left.\pi\right|_{X^{\circ}}$. For any non-zero section $t \in H^{0}\left(\mathrm{Gr}, V \otimes \mathcal{O}_{\mathrm{Gr}}\right)=V$

$$
\Pi \circ t \in H^{0}(\mathrm{Gr}, \mathcal{Q})
$$

cuts out a smooth locus $Z(\Pi \circ t)$ of codimension 2. By Kleiman-Bertini [Har3, Thm. III.10.8], for generic $t$ we have:

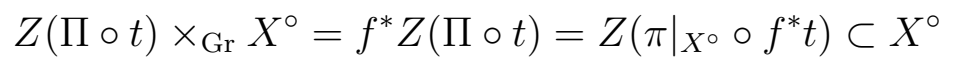

is empty or has codimension 2. The empty case can be ruled out 6 Dualizing $\left.\pi\right|_{X^{\circ}} \circ f^{*} t \in H^{0}\left(X^{\circ}, \mathcal{R}^{\prime *}\right)$ produces a cosection $\left.\mathcal{R}^{\prime}\right|_{X^{\circ}} \rightarrow \mathcal{O}_{X^{\circ}}$ cutting out a 1 dimensional closed subscheme. Since $\mathcal{R}^{\prime}$ is reflexive, this extends to a cosection on $X$ cutting out a 1 -dimensional closed subscheme 7

\section{Moduli SPACES}

2.1. Serre Correspondence. We start by recalling the Serre correspondence Har1, Har2]. For a smooth projective 3-fold $X$, the Serre correspondence gives a bijection between rank 2 locally free sheaves on $X$ with section and certain lci curves on $X$ [Har1]. R. Hartshorne extended this to reflexive sheaves [Har2].

Theorem 2.1 (Hartshorne-Serre correspondence). Let $X$ be a smooth projective 3-fold and $L$ a line bundle on $X$ satisfying $H^{1}(L)=H^{2}(L)=0$. Then there exists a bijective correspondence between:

- Pairs $(\mathcal{R}, \sigma)$, where $\mathcal{R}$ is a rank 2 reflexive sheaf on $X$ with $\operatorname{det}(\mathcal{R}) \cong$ $L$ and $\sigma: \mathcal{R} \rightarrow \mathcal{O}_{X}$ a cosection cutting out a 1-dimensional closed subscheme.

- Pairs $(C, \xi)$, where $C \subset X$ is a Cohen-Macaulay curve which is generically lci and $\xi: \mathcal{O}_{X} \rightarrow \omega_{C} \otimes \omega_{X}^{-1} \otimes L$ has 0-dimensional cokernel.

\footnotetext{
${ }^{6}$ In the empty case, we get a surjective morphism $\left.\mathcal{R}^{\prime}\right|_{X^{\circ}} \rightarrow \mathcal{O}_{X^{\circ}}$. Since $\mathcal{R}^{\prime}$ is reflexive, it lifts to a surjection $\mathcal{R}^{\prime} \rightarrow \mathcal{O}_{X}$. Its kernel is easily seen to be a line bundle by [HL, Prop. 1.1.6, 1.1.10] and hence $\mathcal{R}^{\prime}$ is locally free, contradicting the assumption that $\mathcal{R}^{\prime}$ is singular.

${ }^{7}$ Kleiman-Bertini also gives that the cosection can be taken to cut out a reduced curve.
} 
In this theorem, $\omega_{C} \otimes \omega_{X}^{-1} \otimes L$ is a 1-dimensional pure sheaf. This can be seen by noting $\omega_{C} \cong \mathscr{E} x t^{2}\left(\mathcal{O}_{C}, \omega_{X}\right)$ [Eis, Thm. 21.5] and using [HL, p. 6]. Hence $\left(\omega_{C} \otimes \omega_{X}^{-1} \otimes L, \xi\right)$ is a PT pair in the sense of [PT1]. We introduce some notation. For any sheaf $\mathcal{E}$ on $X$ with support of codimension $c$ and any line bundle $M$ on $X$

$$
(\mathcal{E})_{M}^{D}:=\mathscr{E} x t^{c}(\mathcal{E}, M)
$$

Then $(\mathcal{E})_{\omega_{X}}^{D}$ is the usual notion of a dual sheaf $\mathcal{E}^{D}$ of [HL, Def. 1.1.7]. Moreover

$$
\omega_{C} \otimes \omega_{X}^{-1} \otimes L \cong\left(\mathcal{O}_{C}\right)_{L}^{D}
$$

We now briefly recall how the Serre correspondence works (following Har2 which discusses the case $X=\mathbb{P}^{3}$ but straightforwardly generalizes). Suppose we are given data $(\mathcal{R}, \sigma)$ as in $(1)$. Then the cosection induces a surjection $\mathcal{R} \rightarrow I_{C}$ and a short exact sequence

$$
0 \longrightarrow L \longrightarrow \mathcal{R} \longrightarrow I_{C} \longrightarrow 0
$$

where the kernel $L \cong \operatorname{det}(\mathcal{R})$ is a line bundle. See [Har2] for details. Therefore we obtain an extension $\xi \in \operatorname{Ext}^{1}\left(I_{C}, L\right)$. Note that $\mathcal{R}$ is locally free outside a 0 -dimensional closed subscheme so $C$ is generically lci. Outside the lci locus $C$ is Cohen-Macaulay. Using the short exact sequence

$$
0 \longrightarrow I_{C} \longrightarrow \mathcal{O}_{X} \longrightarrow \mathcal{O}_{C} \longrightarrow 0
$$

we obtain isomorphisms

$$
\mathscr{E} x t^{1}\left(I_{C}, L\right) \cong \mathscr{E} x t^{2}\left(\mathcal{O}_{C}, L\right) \cong \omega_{C} \otimes \omega_{X}^{-1} \otimes L=:\left(\mathcal{O}_{C}\right)_{L}^{D}
$$

where we used definition (5). Using the local-to-global spectral sequence and $H^{1}(L)=H^{2}(L)=0$, we deduce

$$
\operatorname{Ext}^{1}\left(I_{C}, L\right) \cong H^{0}\left(\mathscr{E} x t^{1}\left(I_{C}, L\right)\right) \cong H^{0}\left(\left(\mathcal{O}_{C}\right)_{L}^{D}\right) .
$$

This gives the section $\xi: \mathcal{O}_{X} \rightarrow\left(\mathcal{O}_{C}\right)_{L}^{D}$. Applying $\mathscr{H} o m(\cdot, L)$ to (6) gives

$$
0 \longrightarrow L \longrightarrow \mathcal{R}^{*} \otimes L \longrightarrow \mathcal{O}_{X} \stackrel{\delta}{\longrightarrow} \mathscr{E} x t^{1}\left(I_{C}, L\right) \longrightarrow \mathscr{E} x t^{1}(\mathcal{R}, L) \longrightarrow 0,
$$

where $\delta$ sends 1 to $\xi$. By a slight abuse of notation, we denote $\delta$ by $\xi$ as well. Finally, $\mathscr{E} x t^{1}(\mathcal{R}, L)$ is a 0 -dimensional sheaf which is supported on the locus where $\mathcal{R}$ is not locally free and the length of this sheaf equals $c_{3}(\mathcal{R})$ (see [Har2] for details). We indeed obtain a PT pair $\left(\left(\mathcal{O}_{C}\right)_{L}^{D}, \xi\right)$, which we refer to as a Hartshorne PT pair. This shows how to go from (1) to (2) in Theorem 2.1. The way backwards is explained in [Har2]. 
2.2. Moduli of sheaves. Our initial moduli space is $\operatorname{Quot}(\mathcal{R}, n)$ of the introduction. We also define

$$
\operatorname{Quot}(\mathcal{R}):=\bigsqcup_{n=0}^{\infty} \operatorname{Quot}(\mathcal{R}, n) .
$$

Set theoretically one can write $\operatorname{Quot}(\mathcal{R})$ as

$$
\bigsqcup_{\mathcal{Q} \in \mathcal{T}} \operatorname{Hom}(\mathcal{R}, \mathcal{Q})^{\text {onto }} / \sim
$$

where $\mathcal{T}$ denotes the stack of all 0 -dimensional sheaves on $X$, "onto" refers to the subset of surjective maps in

$$
\operatorname{Hom}(\mathcal{R}, \mathcal{Q}) \cong \operatorname{Ext}^{1}(\mathcal{R}, \mathcal{Q}[-1]),
$$

and the equivalence $\sim$ is induced from the automorphisms of $\mathcal{Q}$. In Section 2.4 we define the "PT analog" of $\operatorname{Quot}(\mathcal{R})((10)$ below $)$

$$
\bigsqcup_{\mathcal{Q} \in \mathcal{T}} \operatorname{Ext}^{2}(\mathcal{Q}, \mathcal{R})^{\text {pure }} / \sim
$$

where

$$
\operatorname{Ext}^{2}(\mathcal{Q}, \mathcal{R}) \cong \operatorname{Ext}^{1}(\mathcal{Q}[-1], \mathcal{R})
$$

2.3. Ext groups. Although $\operatorname{Ext}^{1}(\mathcal{R}, \mathcal{Q}[-1])$ and $\operatorname{Ext}^{1}(\mathcal{Q}[-1], \mathcal{R})$ can jump, their difference cannot. This is the analog of [ST, Lem. 4.10]:

Lemma 2.2. For any rank 2 reflexive sheaf $\mathcal{R}$ and 0 -dimensional sheaf $\mathcal{Q}$ on a smooth projective 3-fold $X$, the only Ext groups between $\mathcal{R}$ and $\mathcal{Q}[-1]$ are $\operatorname{Ext}^{1}(\mathcal{R}, \mathcal{Q}[-1]), \operatorname{Ext}^{1}(\mathcal{Q}[-1], \mathcal{R})$. Moreover

$$
\operatorname{dim} \operatorname{Ext}^{1}(\mathcal{R}, \mathcal{Q}[-1])-\operatorname{dim} \operatorname{Ext}^{1}(\mathcal{Q}[-1], \mathcal{R})=\chi(\mathcal{R}, \mathcal{Q})=2 \ell(\mathcal{Q}),
$$

where $\ell(\mathcal{Q})$ denotes the length of $\mathcal{Q}$.

Proof. Since $\mathcal{R}$ is reflexive, it has homological dimension $\leq 1$ ([Har2, Prop. 1.3] and Auslander-Buchsbaum). Hence $\operatorname{Ext}^{\geq 2}(\mathcal{R}, \mathcal{Q})=0$ and

$$
\begin{aligned}
& 2 \ell(\mathcal{Q})=\chi(\mathcal{R}, \mathcal{Q})=\operatorname{dim} \operatorname{Hom}(\mathcal{R}, \mathcal{Q})-\operatorname{dim}_{\operatorname{Ext}^{1}(\mathcal{R}, \mathcal{Q})} \\
& =\operatorname{dim} \operatorname{Ext}^{1}(\mathcal{R}, \mathcal{Q}[-1])-\operatorname{dim} \operatorname{Ext}^{2}(\mathcal{Q}, \mathcal{R})
\end{aligned}
$$

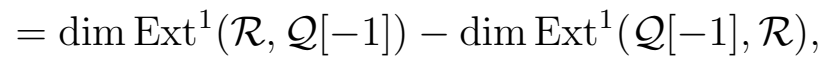

where we use Serre duality and $\mathcal{Q} \otimes \omega_{X}^{-1} \cong \mathcal{Q}$ since $\mathcal{Q}$ is 0 -dimensional. 
2.4. Moduli of PT pairs. In this section we fix data $(\mathcal{R}, \sigma)$ or, equivalently, $\left(\left(\mathcal{O}_{C}\right)_{L}^{D}, \xi\right)$ as in the Serre correspondence. For $\mathcal{Q} \in \mathcal{T}$, we want to define $\operatorname{Ext}^{2}(\mathcal{Q}, \mathcal{R})^{\text {pure }} \subset \operatorname{Ext}^{2}(\mathcal{Q}, \mathcal{R})$. In the rank one case of Stoppa-Thomas [ST]

$$
\operatorname{Ext}^{1}\left(\mathcal{Q}[-1], I_{C}\right) \cong \operatorname{Ext}^{2}\left(\mathcal{Q}, I_{C}\right) \cong \operatorname{Ext}^{1}\left(\mathcal{Q}, \mathcal{O}_{C}\right),
$$

where the second isomorphism is induced by (7). Elements of $\operatorname{Ext}^{1}\left(\mathcal{Q}, \mathcal{O}_{C}\right)$ are short exact sequences

$$
0 \rightarrow \mathcal{O}_{C} \rightarrow F \rightarrow \mathcal{Q} \rightarrow 0,
$$

where $F$ is necessarily 1-dimensional. Define $\operatorname{Ext}^{1}\left(\mathcal{Q}, \mathcal{O}_{C}\right)^{\text {pure }}$ as the locus of extensions for which $F$ is pure. Then elements of $\operatorname{Ext}^{1}\left(\mathcal{Q}, \mathcal{O}_{C}\right)^{\text {pure }}$ are exactly the PT pairs of [PT1] for which the underlying Cohen-Macaulay support curve is $C$.

Lemma 2.3. Let $\mathcal{R}$ be a rank 2 reflexive sheaf on a smooth projective 3-fold $X$ and let $\sigma: \mathcal{R} \rightarrow \mathcal{O}_{X}$ be a cosection cutting out a 1-dimensional closed subscheme $C \subset X$. Then for any 0 -dimensional sheaf $\mathcal{Q}$ on $X$, there exists a natural inclusion

$$
\operatorname{Ext}^{1}(\mathcal{Q}[-1], \mathcal{R}) \hookrightarrow \operatorname{Ext}^{1}\left(\mathcal{Q}[-1], I_{C}\right) .
$$

Proof. This follows by applying $\operatorname{Hom}(\mathcal{Q}, \cdot)$ to the short exact sequence (6)

$$
\cdots \longrightarrow \operatorname{Ext}^{2}(\mathcal{Q}, L) \longrightarrow \operatorname{Ext}^{2}(\mathcal{Q}, \mathcal{R}) \longrightarrow \operatorname{Ext}^{2}\left(\mathcal{Q}, I_{C}\right) \longrightarrow \cdots,
$$

where $\operatorname{Ext}^{2}(\mathcal{Q}, L) \cong \operatorname{Ext}^{1}(L, \mathcal{Q})^{*} \cong H^{1}(\mathcal{Q})^{*} \cong 0$ since $\mathcal{Q}$ is 0 -dimensional.

Using the embedding of Lemma 2.3, we can view

$$
\operatorname{Ext}^{2}(\mathcal{Q}, \mathcal{R}) \subset \operatorname{Ext}^{1}\left(\mathcal{Q}, \mathcal{O}_{C}\right)
$$

as a sublocus and define

$$
\operatorname{Ext}^{2}(\mathcal{Q}, \mathcal{R})^{\text {pure }}:=\operatorname{Ext}^{2}(\mathcal{Q}, \mathcal{R}) \cap \operatorname{Ext}^{1}\left(\mathcal{Q}, \mathcal{O}_{C}\right)^{\text {pure }}
$$

Note that this definition depends not only on $\mathcal{R}$ but also on the chosen cosection $\sigma: \mathcal{R} \rightarrow \mathcal{O}_{X}$. At the level of sets we consider

$$
\bigsqcup_{\mathcal{Q} \in \mathcal{T}} \operatorname{Ext}^{2}(\mathcal{Q}, \mathcal{R})^{\text {pure }} / \sim
$$

where again the equivalence $\sim$ is induced from the automorphisms of $\mathcal{Q}$. By construction, this is a sublocus of the moduli space of all PT pairs. We will now prove that the points of (10) are in bijective correspondence with the closed points of a certain Quot scheme of a 0-dimensional sheaf. First a useful observation: 
Remark 2.4. Let $\mathcal{Q}$ be any 0-dimensional sheaf on $X, F$ any 1-dimensional sheaf on $X$, and $L$ any line bundle on $X$. Then $\mathcal{Q}$ is automatically pure and $F$ is pure if and only if $\mathscr{E} x t^{3}\left(F, \mathcal{O}_{X}\right)=0$ by [HL, Prop. 1.1.10]. Suppose $F$ is pure then, again by [HL, Prop. 1.1.10],

$$
\left(F_{L}^{D}\right)_{L}^{D} \cong F,\left(\mathcal{Q}_{L}^{D}\right)_{L}^{D} \cong \mathcal{Q}
$$

Suppose we are given any PT pair

$$
0 \longrightarrow \mathcal{O}_{C} \longrightarrow F \longrightarrow \mathcal{Q} \longrightarrow 0 \text {. }
$$

Then dualizing using [HL, Prop. 1.1.6] gives a short exact sequence

$$
0 \longrightarrow F_{L}^{D} \longrightarrow\left(\mathcal{O}_{C}\right)_{L}^{D} \longrightarrow \mathcal{Q}_{L}^{D} \longrightarrow 0
$$

where $F_{L}^{D}$ is pure and $\mathcal{Q}_{L}^{D}$ is 0-dimensional. When $F=\left(\mathcal{O}_{C}\right)_{L}^{D}$, e.g. when $(F, s)$ is a Hartshorne PT pair, (13) defines a PT pair as well by (11). So the dual of a Hartshorne PT pair $\left(\left(\mathcal{O}_{C}\right)_{L}^{D}, \xi\right)$ is a PT pair $\left(\left(\mathcal{O}_{C}\right)_{L}^{D}, \xi_{L}^{D}\right)$. In general, dualizing (13) again gives back (12). Hence the operation $(\cdot)_{L}^{D}$ is reversible on PT pairs and defines an involution on PT pairs with $F=\left(\mathcal{O}_{C}\right)_{L}^{D}$.

Theorem 2.5. Let $\mathcal{R}$ be a rank 2 reflexive sheaf on a smooth projective 3fold $X$. Suppose $H^{1}(\operatorname{det}(\mathcal{R}))=H^{2}(\operatorname{det}(\mathcal{R}))=0$ and $\sigma: \mathcal{R} \rightarrow \mathcal{O}_{X}$ is a cosection cutting out a 1-dimensional closed subscheme $C \subset X$. Then the elements of (10) are in natural bijective correspondence with the closed points of $\operatorname{Quot}\left(\mathscr{E} x t^{1}\left(\mathcal{R}, \mathcal{O}_{X}\right)\right)$.

Proof. Step 1: We are given the short exact sequence

$$
0 \longrightarrow L \longrightarrow \mathcal{R} \longrightarrow I_{C} \longrightarrow 0
$$

and the corresponding Hartshorne PT pair $\left(\left(\mathcal{O}_{C}\right)_{L}^{D}, \xi\right)$. Let $\mathcal{Q} \in \mathcal{T}$. An element of $\operatorname{Ext}^{1}(\mathcal{Q}[-1], \mathcal{R})$ corresponds to an exact triangle

$$
\mathcal{R} \rightarrow A^{\bullet} \rightarrow \mathcal{Q}[-1]
$$

Then the image under the natural inclusion $\operatorname{Ext}^{1}(\mathcal{Q}[-1], \mathcal{R}) \subset \operatorname{Ext}^{1}\left(\mathcal{Q}[-1], I_{C}\right)$ of Lemma 2.3 is the induced third column of the following diagram

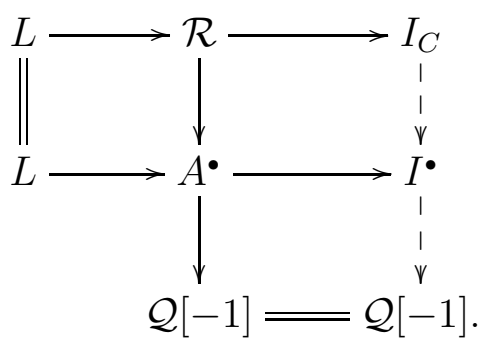


More precisely, an element $I_{C} \rightarrow I^{\bullet} \rightarrow \mathcal{Q}[-1]$ of $\operatorname{Ext}^{1}\left(\mathcal{Q}[-1], I_{C}\right)$ lies in the image of the natural inclusion of Lemma 2.3 if and only if there exists a map $I^{\bullet} \rightarrow L[1]$ such that the following diagram (not an exact triangle) commutes

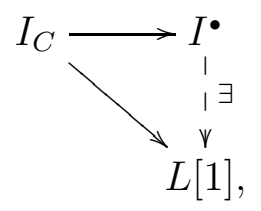

where the diagonal map comes from (14). This can be rephrased: the element $I_{C} \rightarrow I^{\bullet} \rightarrow \mathcal{Q}[-1]$ gives rise to an exact sequence $\operatorname{Ext}^{1}\left(I^{\bullet}, L\right) \rightarrow \operatorname{Ext}^{1}\left(I_{C}, L\right) \rightarrow$ $\operatorname{Ext}^{3}(\mathcal{Q}, L)$ and the above condition is equivalent to

$$
\xi \in \operatorname{im}\left(\operatorname{Ext}^{1}\left(I^{\bullet}, L\right) \rightarrow \operatorname{Ext}^{1}\left(I_{C}, L\right)\right),
$$

where $\xi \in \operatorname{Ext}^{1}\left(I_{C}, L\right)$ is the given extension (14). By exactness, this is equivalent to

$$
\xi \in \operatorname{ker}\left(\operatorname{Ext}^{1}\left(I_{C}, L\right) \rightarrow \operatorname{Ext}^{3}(\mathcal{Q}, L)\right)
$$

Next we note that $\mathscr{E} x t^{i}\left(I_{C}, L\right) \cong \mathscr{E} x t^{i+1}\left(\mathcal{O}_{C}, L\right)$ for all $i>0$. Moreover, since $C$ is Cohen-Macaulay, $\mathscr{E} x t^{i}\left(\mathcal{O}_{C}, L\right)=0$ unless $i=2$. By the local-to-global spectral sequence and $H^{1}(L)=H^{2}(L)=0$

$$
\begin{aligned}
\operatorname{Ext}^{1}\left(I_{C}, L\right) & \cong H^{0}\left(\mathscr{E} x t^{1}\left(I_{C}, L\right)\right), \\
\operatorname{Ext}^{3}(\mathcal{Q}, L) & \cong H^{0}\left(\mathscr{E} x t^{3}(\mathcal{Q}, L)\right) .
\end{aligned}
$$

Hence an element $I_{C} \rightarrow I^{\bullet} \rightarrow \mathcal{Q}[-1]$ of $\operatorname{Ext}^{1}\left(\mathcal{Q}[-1], I_{C}\right)$ lies in the image of the natural inclusion of Lemma 2.3 if and only if the composition

$$
\mathcal{O}_{X} \stackrel{\xi}{\longrightarrow} \mathscr{E} x t^{1}\left(I_{C}, L\right) \longrightarrow \mathscr{E} x t^{3}(\mathcal{Q}, L)
$$

is zero. Here the map $\mathscr{E} x t^{1}\left(I_{C}, L\right) \rightarrow \mathscr{E} x t^{3}(\mathcal{Q}, L)$ is induced by $I_{C} \rightarrow I^{\bullet} \rightarrow$ $\mathcal{Q}[-1]$. This in turn is equivalent to the existence of a morphism

$$
\operatorname{coker}(\xi)=\mathscr{E} x t^{1}(\mathcal{R}, L) \longrightarrow \mathscr{E} x t^{3}(\mathcal{Q}, L)
$$

for which the triangle in the following diagram commutes

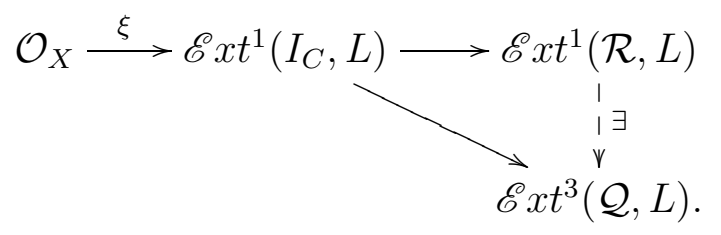

Here the first line is the Hartshorne PT pair (8) 
Step 2: We claim that an element in the image of

$$
\operatorname{Ext}^{1}(\mathcal{Q}[-1], \mathcal{R}) \subset \operatorname{Ext}^{1}\left(\mathcal{Q}[-1], I_{C}\right) \cong \operatorname{Ext}^{1}\left(\mathcal{Q}, \mathcal{O}_{C}\right)
$$

lies in $\operatorname{Ext}^{1}\left(\mathcal{Q}, \mathcal{O}_{C}\right)^{\text {pure }}$ if and only if the arrow in (16) is a surjection. Let $I_{C} \rightarrow I^{\bullet} \rightarrow \mathcal{Q}[-1]$ be an element of $\operatorname{Ext}^{1}\left(\mathcal{Q}[-1], I_{C}\right)$. We get an exact sequence

$$
\cdots \longrightarrow \mathscr{E} x t^{1}\left(I_{C}, L\right) \longrightarrow \mathscr{E} x t^{3}(\mathcal{Q}, L) \longrightarrow \mathscr{E} x t^{2}\left(I^{\bullet}, L\right) \longrightarrow 0,
$$

where we use $\mathscr{E} x t^{2}\left(I_{C}, L\right) \cong \mathscr{E} x t^{3}\left(\mathcal{O}_{C}, L\right)=0$ since $C$ is Cohen-Macaulay. Moreover, writing $I^{\bullet}=\left\{\mathcal{O}_{X} \rightarrow F\right\}$ we have an exact triangle $I^{\bullet} \rightarrow \mathcal{O}_{X} \rightarrow F$. Applying $\mathscr{H} o m\left(\cdot, \mathcal{O}_{X}\right)$ to this exact triangle induces an isomorphism

$$
\mathscr{E} x t^{2}\left(I^{\bullet}, \mathcal{O}_{X}\right) \cong \mathscr{E} x t^{3}\left(F, \mathcal{O}_{X}\right) \text {. }
$$

The claim follows because $F$ is pure if and only if $\mathscr{E} x t^{3}\left(F, \mathcal{O}_{X}\right)=0$.

Step 3: We rephrase the result of Step 2. Given a PT pair $I^{\bullet}=\left\{\mathcal{O}_{X} \rightarrow F\right\}$, we can form the following diagram

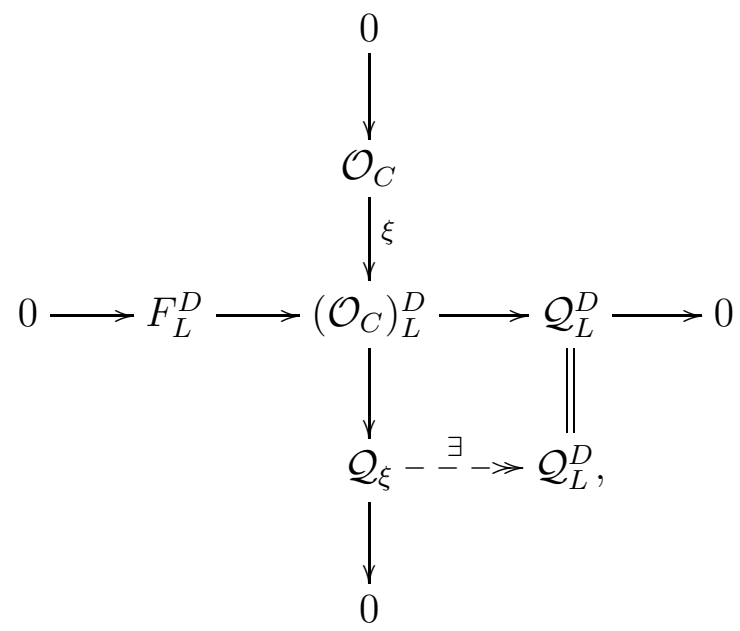

where the middle column is the Hartshorne PT pair and the middle row is the dual of the given PT pair. In particular $\mathcal{Q}_{\xi}:=\operatorname{coker}(\xi)=\mathscr{E} x t^{1}(\mathcal{R}, L)$. Then $I^{\bullet}$ lies in $\operatorname{Ext}^{2}(\mathcal{Q}, \mathcal{R})^{\text {pure }}$ if and only if the indicated surjection exists. The surjection produces the desired element of $\operatorname{Quot}\left(\mathscr{E} x t^{1}(\mathcal{R}, L)\right)$. Since dualizing is a reversible operation (Remark 2.4), we obtain a bijective map to $\operatorname{Quot}\left(\mathscr{E} x t^{1}(\mathcal{R}, L)\right)$.

Remark 2.6 (Sub-PT pairs). One does not actually have to stop at Step 3 of the previous proof. Fix the data $(\mathcal{R}, \sigma)$ as in the Serre Correspondence. Then we are also given a Hartshorne PT pair $\left(\left(\mathcal{O}_{C}\right)_{L}^{D}, \xi\right)$, whose cokernel we denote by $\mathcal{Q}_{\xi}$. As we observed in Remark 2.4, a special feature of the Hartshorne PT 
pair is that its dual is also a PT pair which we denote by $\left(\left(\mathcal{O}_{C}\right)_{L}^{D}, \xi_{L}^{D}\right)$. We introduce the "moduli space" $8 P_{n}(C, \xi)$

$\left\{(F, s):(F, s)\right.$ is a PT pair with $(F, s) \subset\left(\left(\mathcal{O}_{C}\right)_{L}^{D}, \xi_{L}^{D}\right)$ and $\left.\chi(F)=\chi\left(\mathcal{O}_{C}\right)+n\right\}$,

where $(F, s) \subset\left(\left(\mathcal{O}_{C}\right)_{L}^{D}, \xi_{L}^{D}\right)$ means there exists an embedding $F \hookrightarrow\left(\mathcal{O}_{C}\right)_{L}^{D}$ such that the following diagram commutes

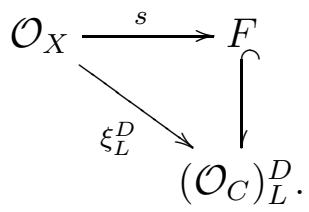

In words: $\bigsqcup_{n} P_{n}(C, \xi)$ consists of sub-PT pairs of the dual of the Hartshorne PT pair. We claim Theorem 1.2 implies:

$$
\sum_{n=0}^{\infty} e(\operatorname{Quot}(\mathcal{R}, n)) q^{n}=M(q)^{2 e(X)} \sum_{n=0}^{\infty} e\left(P_{n}(C, \xi)\right) q^{n}
$$

We now prove this formula. Use the notation of Step 3 of the previous proof. If we have a surjection as indicated, diagram (17) can be completed to

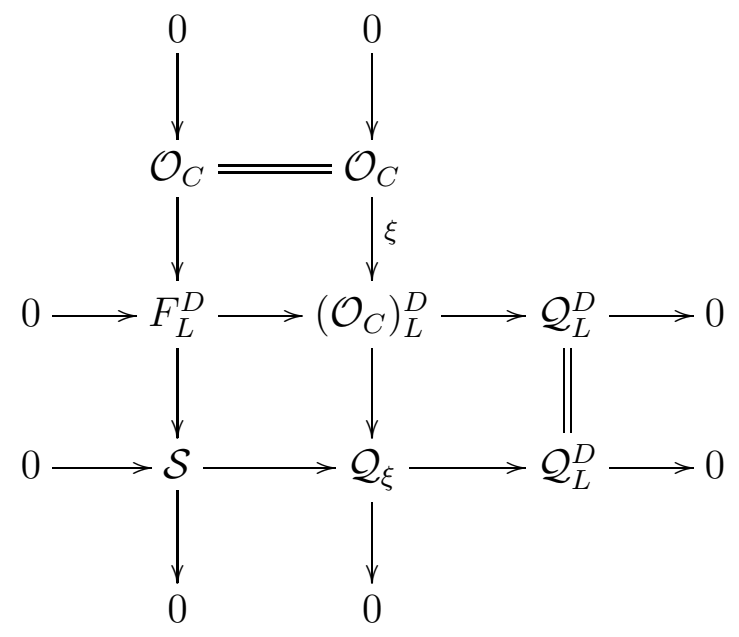

\footnotetext{
${ }^{8}$ Since we only deal with Euler characteristics, we only observe $P_{n}(C, \xi)$ is a constructible subset of the usual space of PT pairs.
} 
where $\mathcal{S}$ denotes the kernel of $\mathcal{Q}_{\xi} \rightarrow \mathcal{Q}_{L}^{D}$. Dualizing diagram (19) yields

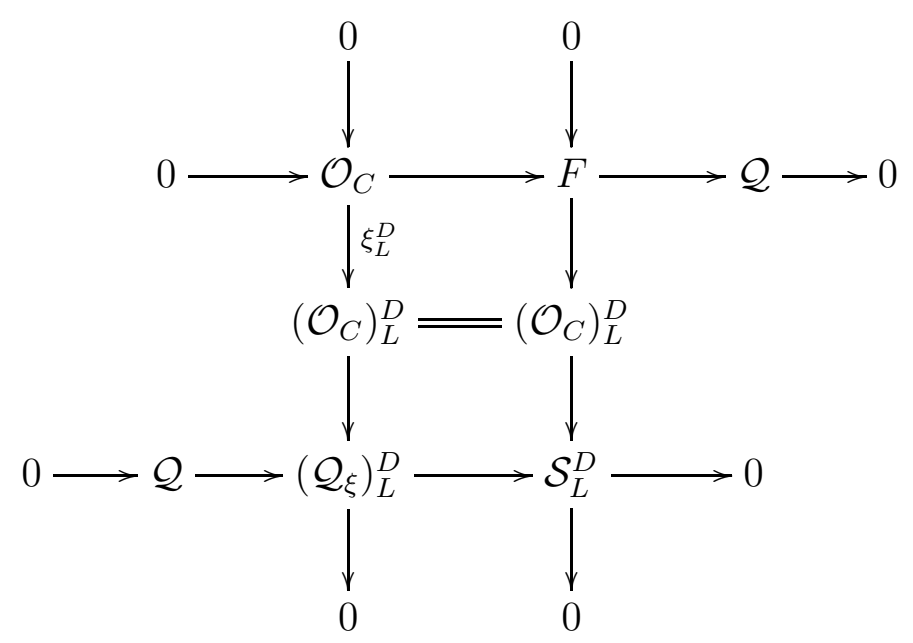

The top square gives rise to the description of $P_{n}(C, \xi)$. Dualizing this diagram we can go back to (19) and (17) (Remark 2.4). Once we establish Theorem 1.2 (done in the next section), formula (18) follows from the bijection

$$
\bigsqcup_{n=0}^{\infty} \operatorname{Quot}\left(\mathscr{E} x t^{1}\left(\mathcal{R}, \mathcal{O}_{X}\right), n\right) \leftrightarrow \bigsqcup_{n=0}^{\infty} P_{n}(C, \xi) .
$$

\section{Hall algebra calculation}

In this section we prove Theorem 1.2 using Theorem 2.5 and a rank 2 version of a Hall algebra calculation of Stoppa-Thomas [ST]. As before, let $X$ be a smooth projective 3 -fold and let $\mathcal{R}$ be a rank 2 reflexive sheaf on $X$ with $H^{1}(\operatorname{det}(\mathcal{R}))=H^{2}(\operatorname{det}(\mathcal{R}))=0$ and a cosection $\sigma: \mathcal{R} \rightarrow \mathcal{O}_{X}$ cutting out a 1-dimensional closed subscheme. We denote by $\mathcal{T}$ the stack of 0 -dimensional sheaves on $X$. We use the following $\mathcal{T}$-stacks:

- $1_{\mathcal{T}}$ is the identity map $\mathcal{T} \rightarrow \mathcal{T}$,

- $\operatorname{Hom}(\mathcal{R}, \cdot)$ is the stack whose fibre over $\mathcal{Q} \in \mathcal{T}$ is $\operatorname{Hom}(\mathcal{R}, \mathcal{Q})$,

- $\operatorname{Hom}(\mathcal{R}, \cdot)^{\text {onto }}$ is the stack whose fibre over $\mathcal{Q} \in \mathcal{T}$ is $\operatorname{Hom}(\mathcal{R}, \mathcal{Q})^{\text {onto }}$,

- $\operatorname{Ext}^{2}(\cdot, \mathcal{R})$ is the stack whose fibre over $\mathcal{Q} \in \mathcal{T}$ is $\operatorname{Ext}^{2}(\mathcal{Q}, \mathcal{R})$,

- $\operatorname{Ext}^{2}(\cdot, \mathcal{R})^{\text {pure }}$ is the stack whose fibre over $\mathcal{Q} \in \mathcal{T}$ is $\operatorname{Ext}^{2}(\mathcal{Q}, \mathcal{R})^{\text {pure }}=\operatorname{Ext}^{2}(\mathcal{Q}, \mathcal{R}) \cap \operatorname{Ext}^{1}\left(\mathcal{Q}, \mathcal{O}_{C}\right)^{\text {pure }} \subset \operatorname{Ext}^{1}\left(\mathcal{Q}, \mathcal{O}_{C}\right)$, where we use the embedding $\operatorname{Ext}^{2}(\mathcal{Q}, \mathcal{R}) \subset \operatorname{Ext}^{1}\left(\mathcal{Q}, \mathcal{O}_{C}\right)$ of Lemma 2.3. Denote by $H(\mathcal{T}):=K(\mathrm{St} / \mathcal{T})$ the Grothendieck group of stacks (locally of finite type and with affine geometric stabilizers) over $\mathcal{T}$. Then $H(\mathcal{T})$ can be endowed with the following product. Let $\mathcal{T}^{2}$ be the stack of short exact 
sequences $0 \rightarrow \mathcal{Q}_{1} \rightarrow \mathcal{Q} \rightarrow \mathcal{Q}_{2} \rightarrow 0$ in $\mathcal{T}$ and let $\pi_{i}$ be the map induced by sending this short exact sequence to $\mathcal{Q}_{i}$. For any two $(\mathcal{T}$-isomorphism classes of) $\mathcal{T}$-stacks $[U \rightarrow \mathcal{T}]$ and $[V \rightarrow \mathcal{T}]$, the product $[U * V \rightarrow \mathcal{T}]$ is defined by the following Cartesian diagram

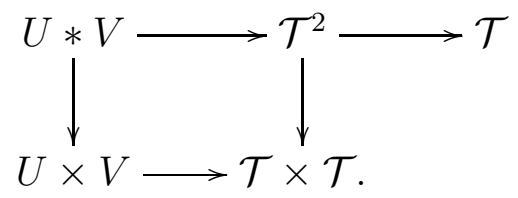

This makes $(H(\mathcal{T}), *)$ into an associative algebra, known as a Joyce's motivic Ringel-Hall algebra. See [ST] and [Bri1, Bri2, Joy1, Joy2, Joy3, Joy4, KS] for details.

Using the inclusion-exclusion principle, we can write $\operatorname{Hom}(\mathcal{R}, \mathcal{Q})^{\text {onto }}$ as

$$
\operatorname{Hom}(\mathcal{R}, \mathcal{Q})-\bigsqcup_{\mathcal{Q}_{1}<\mathcal{Q}} \operatorname{Hom}\left(\mathcal{R}, \mathcal{Q}_{1}\right)+\bigsqcup_{\mathcal{Q}_{1}<\mathcal{Q}_{2}<\mathcal{Q}} \operatorname{Hom}\left(\mathcal{R}, \mathcal{Q}_{1}\right)-\cdots
$$

where $<$ denotes strict inclusion. This leads to the analog in our context of Bridgeland's generalization of Reineke's formula [Bri2]

$$
\operatorname{Hom}(\mathcal{R}, \cdot)=\operatorname{Hom}(\mathcal{R}, \cdot)^{\text {onto }} * 1_{\mathcal{T}}
$$

We recall the following identity from [ST]

$$
\operatorname{Ext}^{1}\left(\cdot, \mathcal{O}_{C}\right)=1_{\mathcal{T}} * \operatorname{Ext}^{1}\left(\cdot, \mathcal{O}_{C}\right)^{\text {pure }} .
$$

Since $\operatorname{Ext}^{2}(\cdot, \mathcal{R}) \subset \operatorname{Ext}^{1}\left(\cdot, \mathcal{O}_{C}\right)($ Lemma 2.3) $)$ "intersecting" this equation with $\operatorname{Ext}^{2}(\cdot, \mathcal{R})$ yields 9

$$
\operatorname{Ext}^{2}(\cdot, \mathcal{R})=1_{\mathcal{T}} * \operatorname{Ext}^{2}(\cdot, \mathcal{R})^{\text {pure }}
$$

Now let

$$
P_{z}(\cdot): H(\mathcal{T}) \longrightarrow \mathbb{Q}(z) \llbracket q \rrbracket
$$

denote the virtual Poincaré polynomial. Here $z$ is the formal variable of $P_{z}$ and $q$ keeps track of an additional grading as follows. Any element $[U \rightarrow \mathcal{T}] \in H(\mathcal{T})$ is locally of finite type and can have infinitely many components. Let $\mathcal{T}_{n} \subset \mathcal{T}$ be the substack of 0 -dimensional sheaves of length $n$ and define

$$
P_{z}(U):=\sum_{n=0}^{\infty} P_{z}\left(U \times_{\mathcal{T}} \mathcal{T}_{n}\right) q^{n}
$$

\footnotetext{
${ }^{9}$ Given $I^{\bullet}=\left\{\mathcal{O}_{X} \rightarrow F\right\} \in \operatorname{Ext}^{1}\left(\cdot, \mathcal{O}_{C}\right)$, modding out by the torsion subsheaf of $F$ leads to an element of $1_{\mathcal{T}} * \operatorname{Ext}^{1}\left(\cdot, \mathcal{O}_{C}\right)^{\text {pure }}$. This map is a geometric bijection. In our context one has to verify that if $I^{\bullet}$ has property (15), then the associated PT pair $\tilde{I}^{\bullet}=\left\{\mathcal{O}_{X} \rightarrow \tilde{F}\right\}$, where $\tilde{F}=F$ torsion also has property (15). This follows from the fact that the natural map $\mathscr{E} x t^{3}(\tilde{\mathcal{Q}}, L) \rightarrow \mathscr{E} x t^{3}(\mathcal{Q}, L)$ is injective, where $\mathcal{Q}, \tilde{\mathcal{Q}}$ are the cokernels of $I^{\bullet}, \tilde{I}^{\bullet}$ respectively.
} 
In fact, $P_{z}(\cdot)$ is a Lie algebra homomorphism to the abelian Lie algebra $\mathbb{Q}(z) \llbracket q \rrbracket$ [ST, Thm. 4.32]. We obtain

$$
\begin{aligned}
P_{z}\left(\operatorname{Ext}^{2}(\cdot, \mathcal{R})^{\text {pure }}\right)\left(z^{2} q\right) & =P_{z}\left(1_{\mathcal{T}}^{-1} * \operatorname{Ext}^{2}(\cdot, \mathcal{R})\right)\left(z^{2} q\right) \\
& =P_{z}\left(\operatorname{Ext}^{2}(\cdot, \mathcal{R}) * 1_{\mathcal{T}}^{-1}\right)\left(z^{2} q\right)
\end{aligned}
$$

where we weigh the component over $\mathcal{T}_{n}$ by $z^{2} q$ instead of $q$ for reasons that will become apparent. We need one more $\mathcal{T}$-stack: $\mathbb{C}^{2 \ell(\cdot)}$, whose fibre over $\mathcal{Q} \in \mathcal{T}$ is $\mathbb{C}^{2 \ell(\mathcal{Q})}$, where $\ell(\mathcal{Q})$ denotes the length of $\mathcal{Q}$. Over strata in $\mathcal{T}$ where $\operatorname{Hom}(\mathcal{R}, \cdot)$ is constant, the stacks

$$
\operatorname{Hom}(\mathcal{R}, \cdot), \operatorname{Ext}^{2}(\cdot, \mathcal{R}) \oplus \mathbb{C}^{2 \ell(\cdot)}
$$

are both Zariski locally trivial of the same rank by Lemma 2.2. We deduce

$$
\begin{aligned}
P_{z}\left(\operatorname{Ext}^{2}(\cdot, \mathcal{R}) * 1_{\mathcal{T}}^{-1}\right)\left(z^{2} q\right) & =P_{z}\left(\left(\operatorname{Ext}^{2}(\cdot, \mathcal{R}) \oplus \mathbb{C}^{2 \ell(\cdot)}\right) *\left(\mathbb{C}^{2 \ell(\cdot)}\right)^{-1}\right)(q) \\
& =P_{z}\left(\operatorname{Hom}(\mathcal{R}, \cdot) *\left(\mathbb{C}^{2 \ell(\cdot)}\right)^{-1}\right)(q)
\end{aligned}
$$

On the other hand

$$
\begin{aligned}
P_{z}\left(\operatorname{Hom}(\mathcal{R}, \cdot)^{\text {onto }}\right)(q) & =P_{z}\left(\operatorname{Hom}(\mathcal{R}, \cdot) * 1_{\mathcal{T}}^{-1}\right)(q) \\
& =P_{z}\left(\left(\operatorname{Hom}(\mathcal{R}, \cdot) *\left(\mathbb{C}^{2 \ell(\cdot)}\right)^{-1}\right) *\left(\mathbb{C}^{2 \ell(\cdot)} * 1_{\mathcal{T}}^{-1}\right)\right)(q)
\end{aligned}
$$

Define $U:=\operatorname{Hom}(\mathcal{R}, \cdot) *\left(\mathbb{C}^{2 \ell(\cdot)}\right)^{-1}$ and $V:=\mathbb{C}^{2 \ell(\cdot)} * 1_{\mathcal{T}}^{-1}$. By [ST, Thm. 4.34], if both $\lim _{z \rightarrow 1} P_{z}(U)$ and $\lim _{z \rightarrow 1} P_{z}(V)$ exist, we have

$$
\lim _{z \rightarrow 1} P_{z}(U * V)=\lim _{z \rightarrow 1} P_{z}(U) \lim _{z \rightarrow 1} P_{z}(V)
$$

In our setting

$$
\begin{array}{r}
\lim _{z \rightarrow 1} P_{z}\left(\mathbb{C}^{2 \ell(\cdot)} * 1_{\mathcal{T}}^{-1}\right)(q)=\lim _{z \rightarrow 1} P_{z}\left(\operatorname{Hom}\left(\mathcal{O}_{X}^{\oplus 2}, \cdot\right)^{\text {onto }}\right)(q)=M(q)^{2 e(X)} \\
\lim _{z \rightarrow 1} P_{z}\left(\operatorname{Hom}(\mathcal{R}, \cdot) *\left(\mathbb{C}^{2 \ell(\cdot)}\right)^{-1}\right)(q)=\lim _{z \rightarrow 1} P_{z}\left(\operatorname{Ext}^{2}(\cdot, \mathcal{R})^{\text {pure }}\right)\left(z^{2} q\right) \\
=\sum_{n=0}^{\infty} e\left(\operatorname{Quot}\left(\mathscr{E} x t^{1}\left(\mathcal{R}, \mathcal{O}_{X}\right), n\right)\right) q^{n} \\
\lim _{z \rightarrow 1} P_{z}(U * V)=\lim _{z \rightarrow 1} P_{z}\left(\operatorname{Hom}(\mathcal{R}, \cdot)^{\text {onto }}\right)(q)=\sum_{n=0}^{\infty} e(\operatorname{Quot}(\mathcal{R}, n)) q^{n}
\end{array}
$$

where the fourth equality follows from Theorem 2.5. This proves Theorem 1.2 . 


\section{TORIC CALCULATION}

In this section we prove Theorem 1.1 by combining Theorem 1.2 with $T$ localization. Since Theorem 1.2 is about smooth projective 3 -folds, we start by compactifying $\mathbb{C}^{3}$. Let $X=\mathbb{P}^{3}$ with homogeneous coordinates $X_{0}, X_{1}, X_{2}, X_{3}$ and let $U_{0}=\left\{X_{0} \neq 0\right\} \cong \mathbb{C}^{3}$.

In chart $U_{0}$, we use coordinates $x_{i}=X_{i} / X_{0}$, for $i=1,2,3$. The action of $T$ on $U_{0}$ is given by $t \cdot x_{i}=t_{i} x_{i}$. We describe a singular rank $2 T$-equivariant reflexive sheaf on $\mathbb{C}^{3}$ by describing the corresponding $\mathbb{Z}^{3}$-graded $\mathbb{C}\left[x_{1}, x_{2}, x_{3}\right]$ module. Here $X(T)=\mathbb{Z}^{3}$ is the character group of $T$. For each $w \in \mathbb{Z}^{3}$ consider the vector space $\mathbb{C} \cdot \mathbf{1}_{w} \oplus \mathbb{C} \cdot \mathbf{2}_{w} \oplus \mathbb{C} \cdot \mathbf{3}_{w}$, where $\mathbf{1}_{w}, \mathbf{2}_{w}, \mathbf{3}_{w}$ denote independent vectors. Choose integers $u_{1}, u_{2}, u_{3} \in \mathbb{Z}, v_{1}, v_{2}, v_{3} \in \mathbb{Z}_{>0}$. The module we will construct has three homogeneous generators of weights

$$
\left(u_{1}, u_{2}, u_{3}\right)+\left(v_{1}, v_{2}, 0\right),\left(u_{1}, u_{2}, u_{3}\right)+\left(v_{1}, 0, v_{3}\right),\left(u_{1}, u_{2}, u_{3}\right)+\left(0, v_{2}, v_{3}\right)
$$

Define

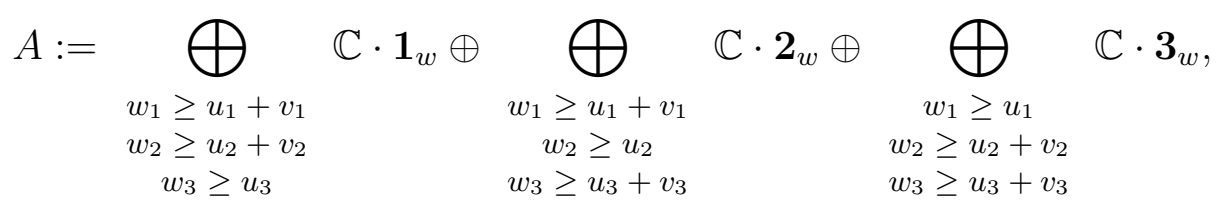

which is a module under $x_{1} \cdot \mathbf{i}_{w}=\mathbf{i}_{w+(1,0,0)}, x_{2} \cdot \mathbf{i}_{w}=\mathbf{i}_{w+(0,1,0)}, x_{3} \cdot \mathbf{i}_{w}=$ $\mathbf{i}_{w+(0,0,1)}$ (adopting notation similar to [PT2]). Define the following $\mathbb{Z}^{3}$-graded $\mathbb{C}\left[x_{1}, x_{2}, x_{3}\right]$-module

$$
R_{0}:=A / \bigoplus_{\begin{array}{l}
w_{1} \geq u_{1}+v_{1} \\
w_{2} \geq u_{2}+v_{2} \\
w_{3} \geq u_{3}+v_{3}
\end{array}} \mathbb{C} \cdot(1,1,1)_{w}
$$

Then $R_{0}$ corresponds to a singular rank $2 T$-equivariant reflexive sheaf $\mathcal{R}_{0}$ on $U_{0} \cong \mathbb{C}^{3}$ generated by three homogeneous generators of weights (22).

Rank $2 T$-equivariant reflexive sheaves on toric 3 -folds and their moduli were studied by the authors in GK1 based on the work of A. Klyachko [Kly1, Kly2. From the toric description ([Kly1, Kly2 or GK1]) it is immediate that all singular rank $2 T$-equivariant reflexive sheaves on $\mathbb{C}^{3}$ arise in the above way (up to $T$-equivariant isomorphism). Moreover, $\mathcal{R}_{0}$ as above extends to a rank $2 T$-equivariant reflexive sheaf $\mathcal{R}$ on $X=\mathbb{P}^{3}$, which is locally free outside $[1: 0: 0: 0]$. This "extension" is unique up to tensoring by a $T$-equivariant 
line bundle on $X$. Since $\left.\mathcal{R}\right|_{U_{i}} \cong \mathcal{O}_{U_{i}} \oplus \mathcal{O}_{U_{i}}$ for $i=1,2,3$, we find

$$
\begin{aligned}
& \sum_{n=0}^{\infty} e(\operatorname{Quot}(\mathcal{R}, n)) q^{n}=\sum_{n=0}^{\infty} e\left(\operatorname{Quot}(\mathcal{R}, n)^{T}\right) q^{n}=\prod_{i=0}^{3} \sum_{n=0}^{\infty} e\left(\operatorname{Quot}\left(\left.\mathcal{R}\right|_{U_{i}}, n\right)^{T}\right) q^{n} \\
& =\left(\sum_{n=0}^{\infty} e\left(\operatorname{Quot}\left(\mathcal{R}_{0}, n\right)^{T}\right) q^{n}\right) \cdot\left(\sum_{n=0}^{\infty} e\left(\operatorname{Quot}\left(\mathcal{O}_{\mathbb{C}^{3}}^{\oplus 2}, n\right)^{T}\right) q^{n}\right)^{3} \\
& =M(q)^{6} \sum_{n=0}^{\infty} e\left(\operatorname{Quot}\left(\mathcal{R}_{0}, n\right)^{T}\right) q^{n}
\end{aligned}
$$

where the first equality is T-localization, the second equality follows from the fact that the cokernels are 0-dimensional, and the fourth equality follows by using the extra scaling $\mathbb{C}^{*}$-action on $\mathcal{O}_{U_{i}} \oplus \mathcal{O}_{U_{i}}$. Therefore in order to prove Theorem 1.1 from Theorem 1.2, we must construct a cosection on $\mathcal{R}$ and prove

$$
\sum_{n=0}^{\infty} e\left(\operatorname{Quot}\left(\mathscr{E} x t^{1}\left(\mathcal{R}, \mathcal{O}_{X}\right), n\right)\right) q^{n}=M(q)^{8} \prod_{i=1}^{v_{1}} \prod_{j=1}^{v_{2}} \prod_{k=1}^{v_{3}} \frac{1-q^{i+j+k-1}}{1-q^{i+j+k-2}}
$$

On $U_{0}$ we choose a generic $T$-equivariant sub-line bundle $L_{0} \subset R_{0}$

$$
L_{0}:=\bigoplus_{\substack{w_{1} \geq u_{1}+v_{1} \\ w_{2} \geq u_{2}+v_{2} \\ w_{3} \geq u_{3}+v_{3}}}\left(\mathbb{C} \cdot(a, b, c)_{w} \oplus \mathbb{C} \cdot(1,1,1)_{w} / \mathbb{C} \cdot(1,1,1)_{w}\right),
$$

where $(a, b, c)$ is sufficiently generic, e.g. $(1,1,0)$ will do. Since the isomorphism type of $\operatorname{Quot}(\mathcal{R}, n)$ does not change when we replace $\mathcal{R}$ by $\mathcal{R} \otimes M$, for any $T$-equivariant line bundle $M$ on $\mathbb{C}^{3}$, we may assume without loss of generality that $u_{1}, u_{2}, u_{3}=0$ in (22). Recall that $v_{1}, v_{2}, v_{3}>0$ remain arbitrary. After this normalization, we obtain a $T$-equivariant isomorphism with an ideal sheaf

$$
R_{0} / L_{0} \cong I_{C_{0}} \cong\left(x_{1}^{v_{1}} x_{2}^{v_{2}}, x_{1}^{v_{1}} x_{3}^{v_{3}}, x_{2}^{v_{2}} x_{3}^{v_{3}}\right),
$$

where $C_{0} \subset U_{0}$ is the union of thickened coordinate axes. Again possibly after replacing $\mathcal{R}$ by $\mathcal{R} \otimes M$, one can easily show from the toric description ([Kly1, Kly2 or GK1]) that $L_{0}$ extends to a global $T$-equivariant sub-line bundle $L \subset \mathcal{R}$, for which there exists a $T$-equivariant short exact sequence

$$
0 \longrightarrow L \longrightarrow \mathcal{R} \longrightarrow I_{C} \longrightarrow 0,
$$

where $C$ is the union of thickenings of the three toric $\mathbb{P}^{1}$ 's meeting in the point $[1: 0: 0: 0]$. Therefore we may apply Theorem 1.2 . 
Finally we observe we are actually in the setting of Remark 1.4. From the way we defined $R_{0}$, it is clear that there exists a $\mathbb{Z}^{3}$-graded short exact sequence

$$
0 \longrightarrow L_{0} \longrightarrow N_{0}^{(1)} \oplus N_{0}^{(2)} \oplus N_{0}^{(3)} \longrightarrow R_{0} \longrightarrow 0,
$$

where

$$
N_{0}^{(1)}:=\bigoplus_{\substack{w_{1} \geq v_{1} \\ w_{2} \geq v_{2} \\ w_{3} \geq 0}} \mathbb{C} \cdot \mathbf{1}_{w}, N_{0}^{(2)}:=\bigoplus_{\substack{w_{1} \geq v_{1} \\ w_{2} \geq 0 \\ w_{3} \geq v_{3}}}^{\bigoplus} \mathbb{C} \cdot \mathbf{2}_{w}, N_{0}^{(3)}:=\bigoplus_{\substack{w_{1} \geq 0 \\ w_{2} \geq v_{2} \\ w_{3} \geq v_{3}}}^{\bigoplus} \mathbb{C} \cdot \mathbf{3}_{w} .
$$

Short exact sequence (24) easily extends to a $T$-equivariant 2-term resolution of $\mathcal{R}$ involving (sums of) line bundles. Taking $\mathscr{H} o m\left(\cdot, \mathcal{O}_{X}\right)$ and using the local description reveals that $\mathscr{E} x t^{1}(\mathcal{R}, L)$ is the structure sheaf of the 0 -dimensional scheme defined by the ideal

$$
I_{\operatorname{Sing}(\mathcal{R})}=\left(x_{1}^{v_{1}}, x_{2}^{v_{2}}, x_{3}^{v_{3}}\right) .
$$

By (3),

$$
\begin{aligned}
\sum_{n=0}^{\infty} e\left(\operatorname{Quot}\left(\mathscr{E} x t^{1}\left(\mathcal{R}, \mathcal{O}_{X}\right), n\right)\right) q^{n} & =\sum_{n=0}^{\infty} e\left(\operatorname{Hilb}^{n}(\operatorname{Sing}(\mathcal{R}))\right) q^{n} \\
& =\sum_{n=0}^{\infty} e\left(\operatorname{Hilb}^{n}(\operatorname{Sing}(\mathcal{R}))^{T}\right) q^{n}
\end{aligned}
$$

The $T$-fixed points of $\operatorname{Hilb}^{n}(\operatorname{Sing}(\mathcal{R}))$ are in bijective correspondence with 3D partitions inside the box $\left[0, v_{1}\right] \times\left[0, v_{2}\right] \times\left[0, v_{3}\right]$. The generating function for these can be found in R. P. Stanley's book [Sta, (7.109)]. Theorem 1.1 follows.

\section{REFERENCES}

[Bri1] T. Bridgeland, An introduction to motivic Hall algebras, Adv. Math. 229 (2012) $102-138$.

[Bri2] T. Bridgeland, Hall algebras and curve counting, JAMS 24 (2011) 969-998.

[Eis] D. Eisenbud, Commutative algebra, with a view towards algebraic geometry, Springer (1999).

[GK1] A. Gholampour and M. Kool, Stable reflexive sheaves and localization, to appear in Jour. of Pure and Applied Alg., arXiv:1308.3688.

[GK2] A. Gholampour and M. Kool, Higher rank sheaves on threefolds and functional equations, arXiv:1706.05246.

[GKY1] A. Gholampour, M. Kool, and B. Young, Rank 2 sheaves on toric 3-folds: classical and virtual counts, arXiv:1509.03536.

[GKY2] A. Gholampour, M. Kool, and B. Young, work in progress.

[Har1] R. Hartshorne, Stable vector bundles of rank 2 on $\mathbb{P}^{3}$, Math. Ann. 238 (1978) 229280.

[Har2] R. Hartshorne, Stable reflexive sheaves, Math. Ann. 254 (1980) 121-176. 
[Har3] R. Hartshorne, Algebraic geometry, Springer-Verlag (1977).

[HL] D. Huybrechts, M. Lehn, The geometry of moduli spaces of sheaves, Cambridge University Press (2010).

[Joy1] D. Joyce, Configurations in abelian categories. I. Basic properties and moduli stacks, Adv. Math. 203 (2006) 194-255.

[Joy2] D. Joyce, Configurations in abelian categories. II. Ringel-Hall algebras, Adv. Math. 210 (2007) 635-706.

[Joy3] D. Joyce, Configurations in abelian categories. III. Stability conditions and identities, Adv. Math. 215 (2007) 153-219.

[Joy4] D. Joyce, Configurations in abelian categories. IV. Invariants and changing stability conditions, Adv. Math. 217 (2008) 125-204.

[Kly1] A. A. Klyachko, Equivariant bundles on toral varieties, Math. USSR Izvestiya 35 (1990) 337-375.

[Kly2] A. A. Klyachko, Vector bundles and torsion free sheaves on the projective plane, preprint Max Planck Institut für Mathematik (1991).

[KS] M. Kontsevich and Y. Soibelman, Stability structures, motivic Donaldson-Thomas invariants and cluster transformations, arXiv:0811.2435.

[PT1] R. Pandharipande and R. P. Thomas, Curve counting via stable pairs in the derived category, Invent. Math. 178 (2009) 407-447. arXiv:0707.2348

[PT2] R. Pandharipande and R. P. Thomas, The 3-fold vertex via stable pairs, Geom. and Topol. 13 (2009) 1835-1876. arXiv:0709.3823

[ST] J. Stoppa and R. P. Thomas, Hilbert schemes and stable pairs: GIT and derived category wall crossing, Bull. Soc. Math. France 139 (2011) 297-339.

[Sta] R. P. Stanley, Enumerative combinatorics, Volume 2, Cambridge Studies in Adv. Math. 62 (2001).

[Tod] Y. Toda, Hall algebras in the derived category and higher rank DT invariants, arXiv:1601.07519,

amingh@umd.edu, m.kool1@uu.nl 